\title{
USING RADIOFREQUENCY ABLATION FOR THE TREATMENT OF LOWER LIMB VARICOSE VEINS
}

Nguyen Van Viet Thanh*, Nguyen Hoai Nam*

Keywords: venous insufficiency, varicose veins, sclerotherapy by radiofrequency ablation.

\section{INTRODUCTION}

Lower limb chronic venous insufficiency is a commonly seen disease which accounted for $40.5 \%$ of people over the age of 50 years old with females having 4.25 higher prevalence compared with males [23]. The lesions could be observed in superficial, perforating, deep veins or all three venous systems in the lower extremities [2]. Superficial veins in particular could be classified in 3 groups: chronic venous insufficiency, varicose veins, and thrombophlebitis. The treatment options of lower limb chronic venous insufficiency in general and chronic venous insufficiency - varicose veins are grouped in two major categories: medication/intervention and surgery. Since 1980s-1990s, endovascular interventions for the treatment of superficial venous insufficiency - varicose veins were introduced and were the new advancement in the treatment of lower limb venous insufficiency disorders $[3,7,11,12,16]$.

\section{DEFINITIONS}

Venous insufficiency in the condition where the valvular systems of the lower limb veins were damaged leading to the reflux of venous blood flow. The lesions could occur in all three venous systems: superficial, perforating, and deep veins $[5,6,9,10]$.

Varicose veins are defined as a dilated and lengthened vein segment that could be seen underlying the skin $[5,6,9,10]$. Varicose veins are divided to primary and secondary disease.
Primary varicose vein is a condition where the dilation of a venous segment causes the dysfunction of venous valves in preventing the reflux of blood return. Conversely, secondary varicose vein is the consequence of valvular insufficiency leading to blood retention and the dilation of a venous segment [1].

\section{HISTORY}

In 1891, D'Arsonval was the first author to describe the use of radiofrequency ablation in medicine. At the beginning, radiofrequency was only implemented in the field of neurology in 1990s. Since then, this measure gradually developed together with the advancement of medicine and science-technology. Since then, more researchers have studied and implemented this method in medicine. In 1999, radiofrequency ablation (RFA) was officially introduced at a conference in New Zealand. Initially, it was only used as a supporting therapy for surgery. This method was later implemented extensively and became an important therapeutic option such as: resection, ablation of cancer tumours, destruction or closed healing of the vessels, treatment of damaged nerve in the body, etc.

Endovascular intervention using radiofrequency ablation (RFA) for the treatment of lower limb venous insufficiency - varicose veins disorders was first introduced in 1998 in Europe. The mechanism of this method is direct thermal effect on the venous wall that leads to sclerosis

*University of medicine and pharmacy at HCM city Scientific director: A/ Prof.|Nguyen Hoai Nam, PhD

Manuscript received: 01/02/2019-Accepted for publication: 23/03/2020

Scientific reviewer: A/ Prof. Dang Ngoc Hung, PhD Prof. Le Ngoc Thanh, PhD 
and contraction of the dilated vein. In 1999, FDA approved RFA as a therapeutic option for venous insufficiency disease in the United States. To date, this technique has been implemented in many countries worldwide including those in the Southeast Asia region such as Thailand, Philippines, and Singapore. Many multi-centre studies have shown that this is an effective, safe method with high cosmetic value compared with conventional surgery. From late 2009 to 2010, Ho Chi Minh Medicine and Pharmacy University Hospital began implementing and successfully applying the RFA technique for the treatment of lower-limb CVI $[11,12,14,16]$.

IV. DIAGNOSIS $[6,10,13,15]$

\section{Signs:}

\section{Types of lower limb varicose veins}

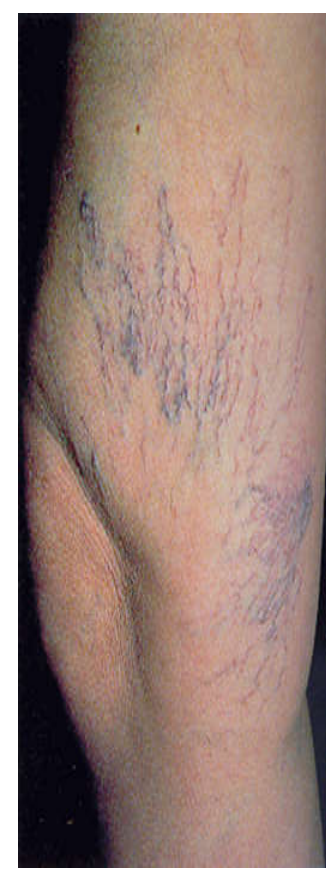

Intradermal varicose veins

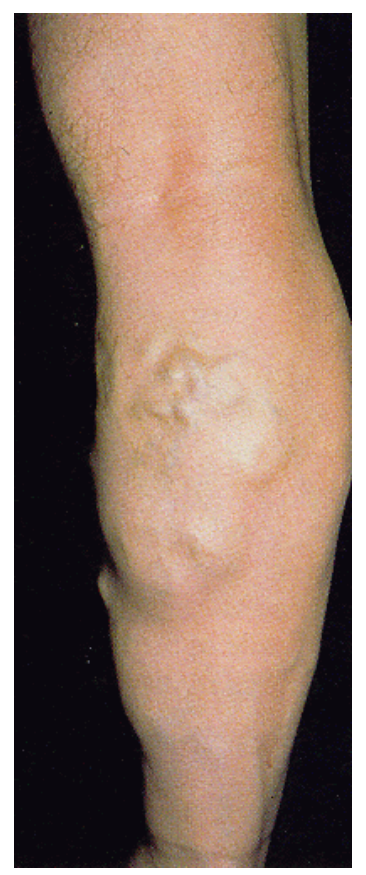

Telangiectasia varicose veins

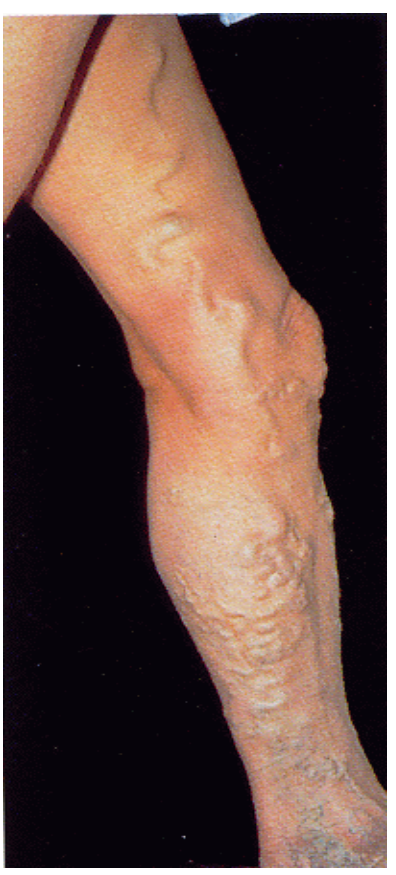

Varicose veins of the Varicose veins of the

great saphenous vein small saphenous vein

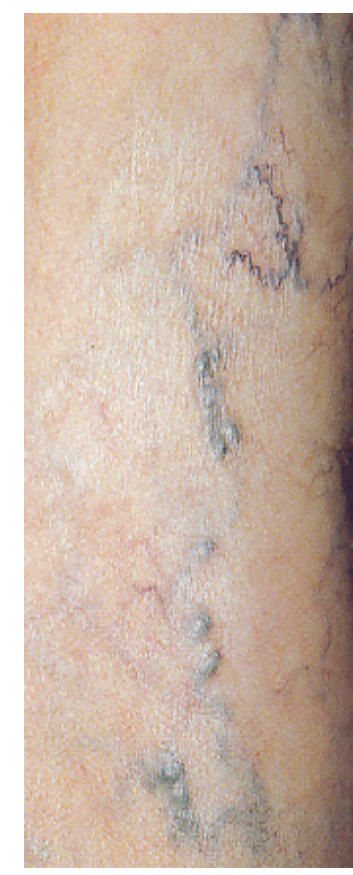

- The patient usually has no or mild symptoms with intradermal, telangiectasia, or mild trunk varicose veins. The chief complain is cosmetic changes.

- Leg pain and heaviness which are worse in the afternoon, numbness, burning, itchy.

- Mild leg oedema, usually around the ankle which is worse in the afternoon or after working, reduced with leg lifting why lying down, cold exposure or by using pressure socks.

Other symptoms possibly associated with chronic CVI such as nocturnal muscle cramp, tired or restlessness legs.

Figure 1: Types of lower limb superficial varicose veins 


\section{Clinical examination}

Patient position: stand on high box and supine, the examiner should pay attention to:

- The presence of varicose veins and their anatomical locations: whether these veins belong to great saphenous vein, small saphenous vein, the secondary, perforating or collateral branches of these veins, etc.

- Leg oedema: uni or bilateral, symmetric or not, location (calf vs. thigh). In case of subtle oedema, a ruler could be used to measure and compare between two legs.

- Skin changes due to malnutrition such as skin discoloration, subcutaneous dermatitis, eczema, skin atrophy, ulcers, etc.

During examination, special manoeuvres could be used to assist the diagnosis and treatment of the disease. The use and interpretation of these manoeuvres are not simple and time consuming. The examiner could apply the following manoeuvres to detect the dysfunction of the superficial venous valvular system:

\section{Schwartz test (tapping test):}

This manoeuvre aims at identifying the valvular dysfunction of the great saphenous vein. The patient stands while the doctor uses two fingers of both hands to place on the dilated vein. The distance between two hands are approximately 10 $\mathrm{cm}$. The upper hand taps on the dilated venous segment while the lower hand feels the impulse of the reflux flow from the upper part. If the lower hand does feel the impulse then the test is positive $(+)$, confirming the diagnosis of great saphenous veins insufficiency.

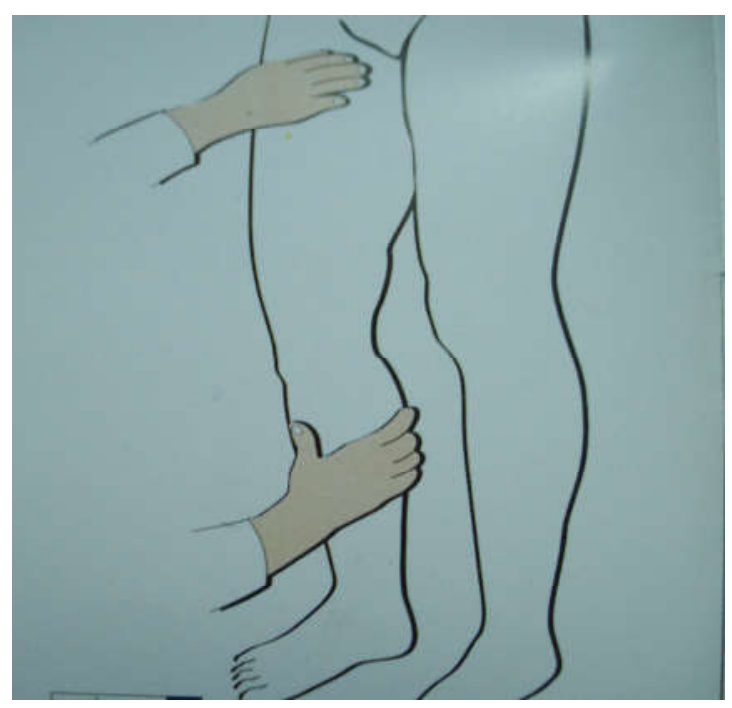

Figure 2: Schwartz test

\section{Coughing test:}

This manoeuvre aims at diagnosis valvular dysfunction of sapheno-femoral vein. The patient stands while the doctor places two fingers at the location where the great saphenous vein joins the femoral vein. A pocket Doppler ultrasound could also be used with the probe placed at the same location mentioned above. Ask the patient to cough, which increases intra-abdominal pressure suddenly, followed by inferior vena cava compression and eventually, reflux flow. The test is $(+)$ when the 
examiner could feel the impulse of reflux flow with his or her hand or a sound of reflux flow could be heard on pocket Doppler ultrasound machine.

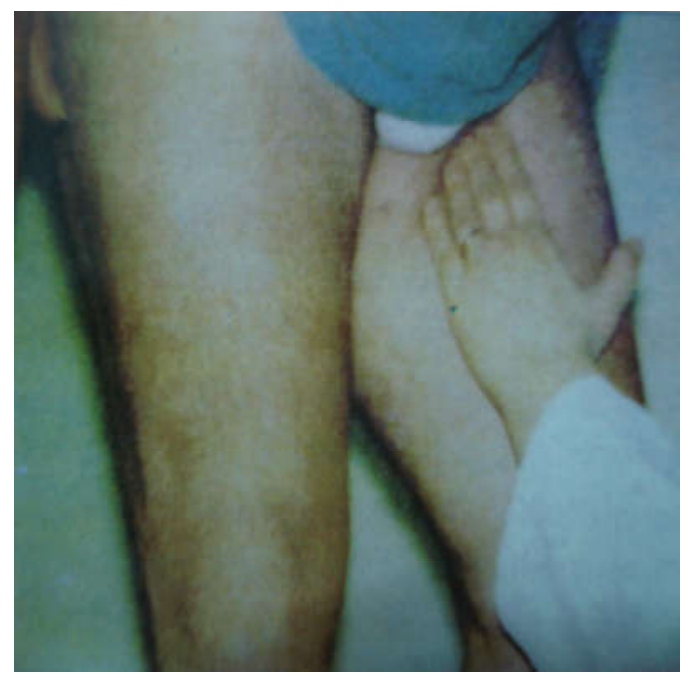

Figure 3: Coughing test

\section{Trendelenburg test:}

This test aims at identifying great saphenous vein and perforating vein insufficiency. The patient is in supine position, legs lifted to drive the blood from great saphenous vein into the deep veins, a tourniquet (venous pressure stitch) is placed at the inguinal fold. Then patient stands up for observation. When the tourniquet is not opened, if the varicose veins re-appear in several seconds $(<30 \mathrm{~s})$ from the lower part of the limb then it is due to the valvular dysfunction of perforating veins. When the tourniquet is opened, if it is great saphenous vein insufficiency then the varicose veins will re-appear from the upper part of the leg within 30 seconds (positive test). The test is negative if the varicose veins re-appear after 30 seconds.

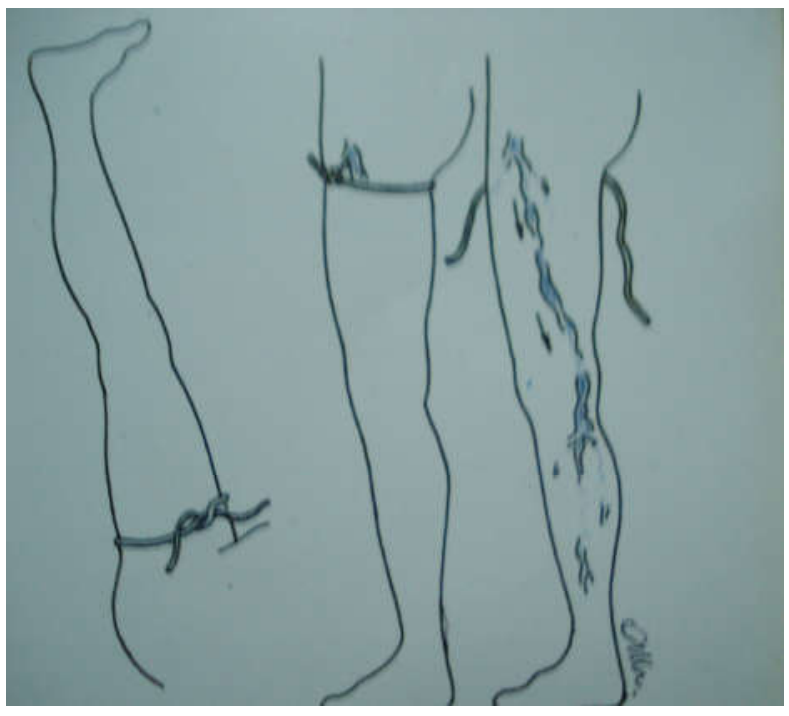

Figure 4: Trendelenburg test 


\section{Paraclinical studies:}

Colour doppler ultrasound: provides us information regarding the function of the valvular system of great saphenous vein, perforating branches and deep veins. This is a non-invasive imaging modality that provides accurate results and can be repeated many times.

Muscle pump ejection fraction testing: evaluates function of the calf muscle pump as well as the valvular systems of deep and superficial veins.

Contrast venography: evaluate the relationship between varicose veins and the deep veins and great saphenous vein. In addition, this test also identifies the location, the valvular function of the perforating branches and where the great and small saphenous veins meet the deep veins.

\section{Classification:}

\section{CEAP classifications}

CEAP stands for: Clinical severity, aEtiology, Anatomy, Pathophysiology.

\section{Clinical classification}

C0: no varicose veins are observed visually or through palpitation

C1: presence of capillary dilation or telangiectasia varicose veins with sizes smaller than $3 \mathrm{~mm}$

$\mathrm{C} 2$ : trunk varicose veins with sizes greater than $3 \mathrm{~mm}$

C3: oedema

C4: skin dystrophy (discolouration, dermatitis, adipose sclerosis, etc.)

C5: skin dystrophy and scar from ulcers

C6: skin dystrophy and ulcers

In combination with:
A: asymptomatic
S: symptomatic

\section{Aetiological classification}

Ec: congenital

Ep: primary

Es: secondary (after thromboembolism)

En: no identified causes

\section{Anatomical classification}

As: superficial veins

Ad: deep veins

Ap: perforating veins

An: not identified

\section{Physio-pathological classification}

Pr: reflux

Po: obstruction

$\mathrm{Pr}+\mathrm{o}$ : reflux and obstruction

Pn: not identified

The advantage of CEAP classification system is that one could identify the aetiology, anatomical locations, physio-pathology and therefore, could draw prognosis and evaluate treatment outcomes. However, this system has a disadvantage that it is quite complicated and could not identify disease severity.

\section{Porter classification}

- Stage 1: No symptoms

- Stage 2: Mild venous insufficiency, ankle oedema, varicose veins

- Stage 3: Moderate venous insufficiency, dermatitis, oedema, adipose sclerosis, varicose veins

- Stage 4: Severe venous insufficiency, apparent oedema, skin dystrophy lesions, ulcers

The advantage of this classification is its 
simplicity, easy to evaluate, classify, and it shows disease severity. However, this classification does not provide clinical, anatomical, physiopathological information like the CEAP classification.

\section{PROGRESSION AND COMPLICATIONS}

Progression [13,15]:

If not treated promptly, the disease will worsen. Based on clinical progression, the disease is classified in two stages:

- Compensated stage: The patient feels strained, heavy and tired in the legs with prolonged standing, there may be mild calf-pedal oedema at the end of a working day that relieves with rest. Lower limb superficial veins are not severely dilated and there are intermittent varicose veins. At the end of this stage, these symptoms worsen, occur more frequently and even at rest with frequent presence of varicose veins.

- Decompensated stage: the patient frequently suffers from leg numbness, itchy at the affected skin, painful walking. The oedema does not relieve with rest. Skin lesions due to dystrophy are present such as dermatitis, sclerosis, ulcers, etc.

\section{Complications [1,5,9]:}

- Thromboembolism: formed at the dilated veins due to blood retention. The thrombi could go to the deep venous system and beyond, resulting in pulmonary embolism which can be lifethreatening.

- Haemorrhage from varicose veins: usually due to trauma, even mild trauma, to the varicose veins, spontaneous haemorrhage is rare. Bleeding could be severe if it is from the main superficial venous trunks.

- Adipose sclerosis: the gradual sclerosis process of the skin and subcutaneous fat tissue. There is no identified aetiology though many mechanisms have been proposed such as increased venous pressure, macrophage capture leading to the release of proteinases, pericapillary fibrin accumulation, etc. This sclerosis is usually clearly identified and located at the low part of the calf and ankle region. The skin becomes stiffening and shiny. This lesion is soft when palpated. The varicose veins could be seen in this area although there is no skin discolouration above the veins while the surrounding area is hyperpigmented. In the acute stage, the skin may look normal and the patient may complain about painful, burning, hot feeling at the lower part of the calf.

- White skin atrophy: a typical skin changes in patients with venous insufficiency, more common in females at the internal ankle and instep. This name originates from the colour and condition of the skin. The typical findings include clear margin hyperpigmented and pale epidermal area due to the lack of capillary that causes the pink colour of the normal skin. Without supplying capillary, the skin suffers from ischemia. Accordingly, the skin tissue is weak and prone to injury. Sometimes this lesion is surrounded by hyperpigmented area and scattered telangiectasia (dilated and lengthened capillaries). Concomitant ulcers are common (1/3 of cases) at white skin atrophy area. There are usually many ulcers with small sizes, very painful and take long time to heal. White skin atrophy can also be an ulcer scar due to healed venous lesions.

- Eczema: Eczema usually develops during the course of venous insufficiency. Capillary disorders due to venous blood retention can be accompanied by eczematous lesions with clear and diffused margin. This phenomenon could be caused by the increased venous pressure, capture and activation of leukocytes. The skin surface is 
usually dry and shiny. The lesion is very itchy and usually associated with secondary neurodermatitis.

Leg ulcers: this is considered the eventual and worst skin lesions of venous insufficiency. Leg ulcers are usually located from the lower part of the calf to the internal ankle and rarely seen on the instep or toes. The onset of ulcer could be subtle or sudden after trauma, haemorrhage due to the rupture of the dilated vein, or skin infection.

\section{TREATMENT USING RADIOFREQUENCY ABLATION}

Acting mechanism: This method alates the tissue using heat caused by the friction between ions inside the tissue under the effect of a alternating current with frequency in the range of radio wave $(200-1.200 \mathrm{MHz})$. The current is transmitted from the generator to the body through a needle electrode and causes heat. The heat caused by friction leads to cellular dehydration and coagulative necrosis of the tissue that need to be ablated [18].

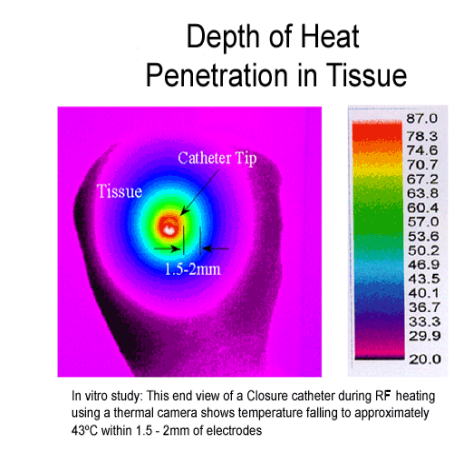

A: The transmission of heat

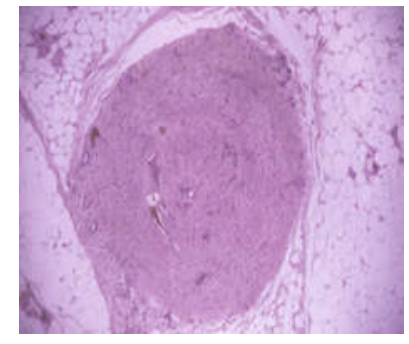

B: Pathological venous changes

Figure 5: The acting mechanism of high frequency wave

Indications and contra-indications of
endovascular laser
Indications
Patients with superficial varicose veins meeting
the following criteria $[14,17,20,21]$ :
- Varicose veins of great saphenous vein and has
reflux flow on doppler ultrasound
- $5 \mathrm{~mm} \leq$ diameter of great saphenous vein trunk $\leq$
$12 \mathrm{~mm}$

\section{Contra-indications}

Patients with the following conditions $[11,14,17,20,21]$ :

- Deep vein thrombosis

- Venous thromboembolism near the arch of great saphenous vein
- Arteriovenous malformation

- The superficial branch is dilated but there is no venous insufficiency or varicose veins of great saphenous vein

- Tortuous great saphenous vein that impedes wire introduction

- Pregnant or breast-feeding women

\section{Preprocedural patient preparation}

Patients indicated for endovascular interventions are prepared the same way with patients undergoing surgery, including:

- Pre-operative laboratory tests

- Careful consultation with patient family and relatives with written informed consent 
- Cleaning the intervened leg

- Drawing interventional map using insoluble ink to identify: location and diameter of the arch of great saphenous vein, the location $2 \mathrm{~cm}$ away from the arch, trunk diameter, reflux flow, needle access location

- Preoperative antibiotics: 30 minutes before procedure
- Establishment of venous lines

\section{Interventional techniques}

\section{Tools}

- Vascular sonogram

- Radiofrequency generator

- Vascular interventional devices.
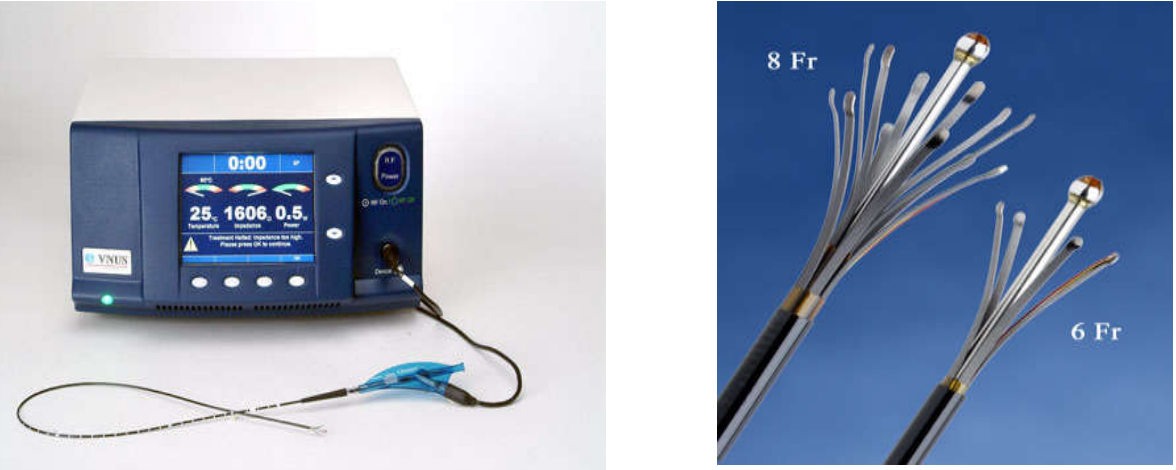

Figure 6: Radiofrequency generator

Positioning the patient [14,17,20,21]

- For great saphenous vein intervention: patient is in supine position, the treated leg is in slight abduction and external rotation position, slightly flexed knee to expose the inside of the thigh

- For small saphenous vein intervention: patient is in prone position, both legs straightly flexed to expose the posterior calf and ham.

\section{Anaesthesia}

- Spinal

- Local

\section{Techniques}

- Prepare the patient similar to surgical patients: sterile the whole length of the leg by betadine, spread the sterile drape

- The doctors wash their hands, wear the sterile gowns and gloves
- Introduce the needle to the saphenous venous under the guidance of ultrasound or Müller method to expose the saphenous vein.

- Use Seldinger technique to introduce the electrode to the marked location (1-2 cm away from the arch). The electrode wire is fixed by a lock. Double-check the location of the wire by ultrasound.

- Inject buffer solution (natriclorua 0.9\%) around the trunk of saphenous vein under ultrasound from distal to proximal. The injection of buffer solution has 3 efefcts. First, it relieves pain. Second, it makes the vein smaller and therefore increases treatment efficacy. Third, it creates a watery environment to protect surrounding veins, reduce heat-related complications to adjacent tissues.

- Based on which generation the genator is, use an energy from $25-30 \mathrm{~W}$, temperature 
from $85^{\circ}-120^{\circ}$. Each intervened segment has a length of $5-7 \mathrm{~cm}$. Procedural time for each venous segment is $20 \mathrm{~s}$.

- After the procedure, patient had his or her leg covered with pressure bandage or wears venous socks in 7 days and can be discharged as soon as 2 hours after intervention.

- The patient had a follow-up visit at 1 week. The remaining varicose veins were treated by Müller method or ultrasound-guided sclerotherapy.
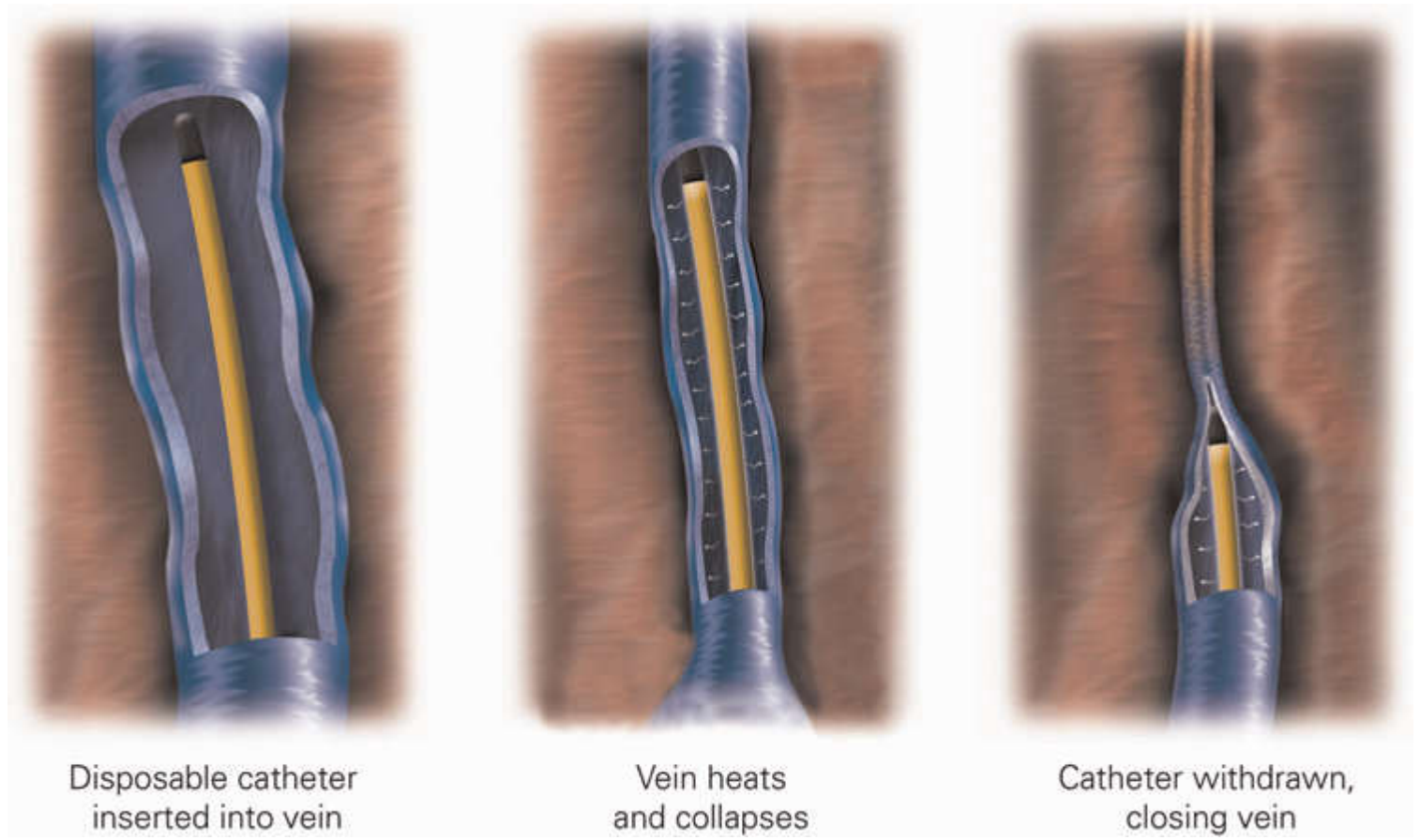

Figure 7: Venous sclerotherapy using radiofrequency ablation

\section{Evaluation of treatment outcome}

\section{Evaluation of treatment outcome based on Mayo}

- Clinic criteria $[4,8,11]$

- Very good: no clinical symptoms, no recurrence of lateral branches

- Good: no clinical symptoms except for recurrence of lateral branches

- Average: clinical symptoms are partly improved and lateral veins are present but no main branch exists

- Failed: the main branch still exists or recurs

Evaluation of laboratory results $[4,8,11]$

- Obstruction of saphenous veins observed on doppler ultrasound
- Reflux flow of saphenous veins observed on doppler ultrasound

\section{Complications:}

- Burn

- Deep vein thrombosis can lead to pulmonary embolism [22]

- Haematoma at the vascular access point

- Infection

\section{Prognosis:}

- High success rate, $96-97 \%$.

- Short hospital length of stay: patients were discharged on the same day

- Rapid recovery: patients could return to normal work within 2 days. 


\section{CONCLUSIONS}

Superficial varicose veins is an increasingly common disease in our country. To date, there are many therapeutic options for this disease. Sclerotherapy using radiofrequency ablation is a minimally invasive, highly effective option with low rate of complications, high cosmetic value and rapid recovery. Together with endovascular laser, radiofrequency ablation are the first-line choices of vascular specialist for the treatment of superficial varicose veins.

\section{REFERENCES}

1. Claudio Allegra (2001), "Venous insufficiency", Discover venous insufficiency disorder, Venous disorders Conference - Ho Chi Minh city Healthworker Training and Education Centre, Servier, HCM city, p 15-24.

2. Claudio Allegra (2001), "Epidemiology of venous disease", Discover venous insufficiency disorder, Venous disorders Conference - Ho Chi Minh city Healthworker Training and Education Centre, Servier, HCM city, p, tr 3-6.

3. Phan Thanh Hai, Ho Khanh Duc, Nguyen Van Viet Thanh (2010), "Treatment of lower limb superficial venous insufficiency by endovascular laser with $810 \mathrm{~nm}$ wavelength diode laser ", HCM city Medical Journal, volume 14 (1), p 168-173.

4. Le Thi Ngoc Hang (2008), Evaluating outcomes of surgical treatment of lower limb variose veins, Resident doctor graduation thesis, HCM Medicine and Pharmacy University, HCM city, p 96-97.

5. Le Nu Thi Hoa Hiep (2003), "Lower limb chronic venous insufficiency: risk factors and indications for surgery", HCM city Medical journal, volume 7, p 97-99.

6. Le Nu Thi Hoa Hiep (2008), "Lower limb superficial venous insufficiency", Surgical treatment of thoracic-cardiovascular diseases, Medicine Publisher, HCM city, p 72-85.

7. Nguyen Hoai Nam (2006), "New therapies for the treatment of chronic venous insufficiency", Updates on treatment of thoracic - vascular diseases", Medicine Publisher, HCM city, p 195-207.

8. Bui Duc Phu, Bui Minh Thanh (2004), "Investigate the application of Stripping surgery for the treatment of lower limb chronic venous insufficiency", Vietnam medical and pharmacy information.

9. Van Tan (2004), "Venous insufficiency and varicose veins", Surgical pathology lecture, Medicine Publisher, HCM city, p 416-439.

10. Van Tan (2007), " Venous insufficiency and varicose veins: pathology and treatment", Surgical treatment lecture, Medicine Publisher, HCM city, p 440-451.

11. Nguyen Van Viet Thanh (2011), "Evaluate the efficacy of treating varicose veins of great saphenous vein by using endovascular laser", Master in Medicine thesis, HCM Medicine and Pharmacy University, HCM, p 90.

12. Bull Phillip (2006), "History of Varicose Vein Surgery", The harley treet vein clinic, London, http://www.dr-bull.com/.

13. D.lafrati Mark, Thomas F. O'Donnell Jr. (2004), "Varicose veins", Haimovici's Vascular Surgery, Blackwell, pp 1058-1073.

14. Gohel, M.S., A.H. Davies (2009), "Radiofrequency ablation for uncomplicated varicose veins", Phlebology, 24 Suppl 1: p. 42-9.

15. Keagy Blair A., Mendes Robert R. (2007), "Superficial Venous Pathology and Therapies", Mastery of Surgery, Lippincott Williams \& Wilkins, Vol 2, pp 2503 - 2531. 